\section{Platelet-derived growth factor receptor $\beta$ activation and regulation in murine myelofibrosis}

\author{
Frederike Kramer, ${ }^{1,2}$ Jens Dernedde, ${ }^{1}$ Artur Mezheyeuski, ${ }^{3}$ Rudolf Tauber, ${ }^{1}$ \\ Patrick Micke ${ }^{3}$ and Kai Kappert ${ }^{1,2,4}$
}

${ }^{1}$ Charité - Universitätsmedizin Berlin, corporate member of Freie Universität Berlin, Humboldt-Universität zu Berlin, and Berlin Institute of Health, Institute of Laboratory Medicine, Clinical Chemistry and Pathobiochemistry, Berlin, Germany; ${ }^{2}$ Charité Universitätsmedizin Berlin, corporate member of Freie Universität Berlin, HumboldtUniversität zu Berlin, and Berlin Institute of Health, Center for Cardiovascular Research (CCR), Berlin, Germany; ' ${ }^{3}$ epertment of Immunology, Genetics and Pathology, Uppsala University, Uppsala, Sweden and ${ }^{4}$ DZHK (German Centre for Cardiovascular Research), partner site Berlin, Berlin, Germany

\section{ABSTRACT}

T There is prevailing evidence to suggest a decisive role for plateletderived growth factors (PDGF) and their receptors in primary myelofibrosis. While PDGF receptor $\beta$ (PDGFR $\beta$ ) expression is increased in bone marrow stromal cells of patients correlating with the grade of myelofibrosis, knowledge on the precise role of PDGFR $\beta$ signaling in myelofibrosis is sparse. Using the Gata- $1^{\text {low }}$ mouse model for myelofibrosis, we applied RNA sequencing, protein expression analyses, multispectral imaging and, as a novel approach in bone marrow tissue, an in situ proximity ligation assay to provide a detailed characterization of PDGFR $\beta$ signaling and regulation during development of myelofibrosis. We observed an increase in PDGFR $\beta$ and PDGF-B protein expression in overt fibrotic bone marrow, along with an increase in PDGFR $\beta$-PDGF-B interaction, analyzed by proximity ligation assay. However, PDGFR $\beta$ tyrosine phosphorylation levels were not increased. We therefore focused on regulation of PDGFR $\beta$ by protein tyrosine phosphatases as endogenous PDGFR $\beta$ antagonists. Gene expression analyses showed distinct expression dynamics among PDGFR $\beta$-targeting phosphatases. In particular, we observed enhanced Tcell protein tyrosine phosphatase protein expression and PDGFR $\beta$-T-cell protein tyrosine phosphatase interaction in early and overt fibrotic bone marrow of Gata-1 $1^{\text {low }}$ mice. In vitro, T-cell protein tyrosine phosphatase (Ptpn2) knockdown increased PDGFR $\beta$ phosphorylation at $Y^{751}$ and $Y^{1021}$, leading to enhanced downstream signaling in fibroblasts. Furthermore, Ptpn2 knockdown cells showed increased growth rates when exposed to low-serum growth medium. Taken together, PDGF signaling is differentially regulated during myelofibrosis. Protein tyrosine phosphatases, which have so far not been examined during disease progression, are novel and hitherto unrecognized components in myelofibrosis.

\section{Introduction}

Primary myelofibrosis (PMF) is a malignant hematologic disorder characterized by the clonal proliferation of hematopoietic stem cells (HSC) in the bone marrow. Patients display symptoms of ineffective hematopoiesis such as anemia, thrombocytopenia and related extramedullary hematopoiesis resulting in splenomegaly. The bone marrow of PMF patients shows dysplastic megakaryocytes, neoangiogenesis and, as a central pathological feature, progressive fibrosis. ${ }^{1}$ The development of myelofibrosis is mainly ascribed to the overproduction of pro-fibrotic cytokines and growth factors by malignant immature cells of the megakaryocytic lineage. As a consequence, fibroblasts proliferate and produce extensive amounts of extracellular matrix (ECM) components, leading to impaired hematopoietic function of the bone marrow. ${ }^{2}$
Ferrata Storti Foundation
Haematologica 2020
Volume 105(8):2083-2094

\section{Correspondence:}

KAI KAPPERT

kai.kappert@charite.de

Received: May 14, 2019.

Accepted: October 29, 2019.

Pre-published: October 31, 2019.

doi:10.3324/haematol.2019.226332

Check the online version for the most updated information on this article, online supplements, and information on authorship \& disclosures: www.haematologica.org/content/105/8/2083

\section{(C)2020 Ferrata Storti Foundation}

Material published in Haematologica is covered by copyright. All rights are reserved to the Ferrata Storti Foundation. Use of published material is allowed under the following terms and conditions:

https://creativecommons.org/licenses/by-nc/4.0/legalcode. Copies of published material are allowed for personal or internal use. Sharing published material for non-commercial purposes is subject to the following conditions: https://creativecommons.org//licenses/by-nc/4.0/legalcode, sect. 3. Reproducing and sharing published material for commercial purposes is not allowed without permission in writing from the publisher. 
Abberantly activated janus kinase-signal transducer and activator of transcription (JAK-STAT) signaling has been identified as a driver of clonal cells in PMF patients. ${ }^{3}$ Somatic mutations in $J A K 2,{ }^{4}$ the thrombopoietin receptor $M P L^{5}$ and $C A L R^{6}$ are the most prevalent genetic aberrations. These, however, are also frequently found in other myeloproliferative neoplasms (MPN), making a definite diagnosis of early PMF problematic. Thus, efforts are directed towards novel and valid diagnostic markers.

Platelet-derived growth factors (PDGF) have been implicated in the progression of bone marrow fibrosis., PDGF-A and -B, as well as PDGF receptor $\alpha$ (PDGFR $\alpha$ ) and PDGF receptor $\beta$ (PDGFR $\beta$ ) expression is increased in the bone marrow of PMF patients, regardless of driver mutations. ${ }^{9,10}$ The PDGF system comprises five dimeric ligands: PDGF-AA, $-\mathrm{AB},-\mathrm{BB},-\mathrm{CC}$ and $-\mathrm{DD} .{ }^{11}$ The two cognate transmembrane receptors, PDGFR $\alpha$ and PDGFR $\beta$, dimerize upon ligand binding and cross-phosphorylate intracellular tyrosine residues. These phosphorylated residues serve as binding sites for downstream signaling components and activate, among others, phospholipase C $\gamma$ (PLC $\gamma$ ), phosphatidyl inositol 3-kinase (PIBK), and JAK-STAT signaling. ${ }^{12} \mathrm{~A}$ large proportion of PDGF derive from megakaryocytes, from where the ligands act on their receptors in a paracrine and autocrine manner. ${ }^{13}$ Whereas PDGFR $\alpha$ binds PDGF-A, -B and -C, PDGFR $\beta$ can only be activated by PDGF-B and -D. Therefore, depending on the ligand dimers, PDGFR $\alpha$ and PDGFR $\beta$ can form homo- and, if co-expressed, heterodimers. ${ }^{14}$ Distinct functions of PDGFR $\alpha$ and PDGFR $\beta$ have been ascribed to the discrete, cell-type specific expression of the receptors. The receptors are predominantly expressed by cells of mesenchymal origin; within the bone marrow, PDGFR $\alpha$ expression is highest in megakaryocytes, whereas PDGFR $\beta$ is almost exclusively expressed in fibroblasts. ${ }^{9,10}$ Therefore, PDGFR $\beta$ has been attributed a major role in the proliferation of bone marrow stromal cells in myelofibrosis. Different mechanisms are involved in the regulation of PDGF signaling, e.g. injury and pro-inflammatory cytokines affect expression of the ligands and receptors. ${ }^{15}$ Furthermore, protein tyrosine phosphatases (PTP) dephosphorylate intracellular tyrosine residues of PDGF receptors and negatively regulate PDGF signaling.

PDGFR $\beta$ expression in activated fibroblasts correlates with the grade of myelofibrosis in the bone marrow of PMF patients. ${ }^{10}$ However, the mechanisms of transformation from malign clonal proliferation of HSC to myelofibrosis and the involvement of the PDGF system are not fully understood. In particular, the expression dynamics of PDGFR $\beta$, the interaction with the ligand PDGF-B, and a possible regulation by PTP during the development of bone marrow fibrosis have not been thoroughly addressed. Using the Gata- $1^{\text {low }}$ mouse model for PMF, this study concentrates on PDGFR $\beta$ and its relevance in stromal cell proliferation. These mice show reduced Gata-1 expression in megakaryocytes, which have a high proliferation rate while remaining immature and releasing reduced platelet numbers. Therefore, Gata- $1^{\text {low }}$ mice develop fibrosis in the bone marrow that resembles the development of myelofibrosis in PMF patients. ${ }^{16}$ For a detailed characterization, we performed whole transcriptome analyses, protein expression and localization analyses in pre-fibrotic, early fibrotic and overt fibrotic bone marrow. Furthermore, we applied a proximity ligation assay (PLA) as a novel, sensitive technique for the quantification of protein expression, interaction of PDGFR $\beta$ with its ligand PDGF-B, as well as PDGFR $\beta$ tyrosine phosphorylation and the interaction of PDGFR $\beta$ with $T$ cell protein tyrosine phosphatase (TC-PTP) for a detailed evaluation of PDGFR $\beta$ activation status in bone marrow fibrosis in situ. Finally, we provide evidence for the regulation of PDGFR $\beta$ by TC-PTP in fibroblasts in vitro.

\section{Methods}

\section{Mouse model}

Gata- $1^{\text {low }}$ mice were purchased from Jackson Laboratory (Bar Harbour, ME, USA) and bred according to standards of the animal facility at the Center for Cardiovascular Research of Charite Universitätsmedizin Berlin (Berlin, Germany). All littermates were genotyped using polymerase chain reaction (PCR) according to the standard protocol provided by Jackson. Hemizygous males and age-matched wild-type (WT) littermates were euthanized at 5, 10 and 15 months of age.

Further material and methods used in this study are described in the Online Supplementary Appendix.

\section{Statistical analysis}

Results presented as boxplots show the median, with whiskers representing minima and maxima; bar graphs show mean and standard deviation. Statistical differences between a Gata- $1^{\text {low }}$ and the age-matched WT control group were determined using unpaired Student $t$-test. For comparison of multiple groups, analysis of variance with post hoc Tukey correction was used. Statistical analyses were performed using GraphPad Prism 6.01 (GraphPad Software Inc., San Diego, CA, USA). $P<0.05$ was considered statistically significant.

\section{Results}

\section{Development of myelofibrosis in the bone marrow of Gata- $1^{\text {low }}$ mice}

We determined different stages of bone marrow fibrosis in a cohort of Gata- $1^{\text {low }}$ mice, which served as time points for all subsequent analyses: 5 months, 10 months and 15 months of age. Mice of all ages were normal in body weight (Figure 1A). As early as 5 months of age, Gata- $1^{\text {low }}$ mice showed a pronounced splenomegaly (Figure 1B), whereas liver weight remained normal at all ages (Figure 1C). Mice displayed time-dependent, progressive anemia (Figure 1D) and a slight increase in leukocytes starting at month 10 (Figure 1E). Platelets were markedly reduced at all ages (Figure 1F). To determine fibrotic stages in Gata$1^{\text {low }}$ mice, we stained murine femoral bone marrow for reticulum fibers (Figure 1I). We did not observe an apparent accumulation of reticulum fibers in the bone marrow of Gata- $1^{\text {low }}$ mice at month 5 , whereas there was a timedependent increased deposition of fibers at month 10 and 15 . Hence, month 5 was defined as a pre-fibrotic, month 10 as an early fibrotic, and month 15 as an overt fibrotic stage in Gata- $1^{\text {low }}$ mice. To monitor the induction of collagen production in the bone marrow of Gata- $1^{\text {low }}$ mice, we analyzed type I collagen Colla1 and type III collagen Col3a1 gene expression by qPCR (Figure $1 \mathrm{G}$ and $\mathrm{H}$ ). We observed a significant decrease in Colla1 gene expression in the pre-fibrotic stage. However, a marked increase in gene expression of the two collagens was detected in early 
fibrotic bone marrow and remained increased in overt fibrotic bone marrow of 15 -month-old Gata- $1^{\text {low }}$ mice.

Differential expression of receptor tyrosine kinases and their ligands in myelofibrosis

Since receptor tyrosine kinases (RTK) and RTK-activating ligands have been attributed an important role in myelofibrosis, we investigated transcriptomic changes by RNA sequencing (RNAseq) analyses with a focus on RTK
(Figure 2A) and their cognate ligands (Figure 2B) Interestingly, a high number of RTK showed a significant increase in gene expression; among which Ptk7, Tie1, Flt1, Fgfr1 and the PDGF receptors Pdgfra and Pdgfrb. Although many ligands were not significantly regulated in early fibrotic bone marrow of Gata-1 $1^{\text {low }}$ mice, we observed an induction of Ptn, Efn61, Pgf, Angpt2, Angpt4, Igf2 and Efna2. To classify transcriptionally up-regulated genes to their biological function, we further performed gene ontology
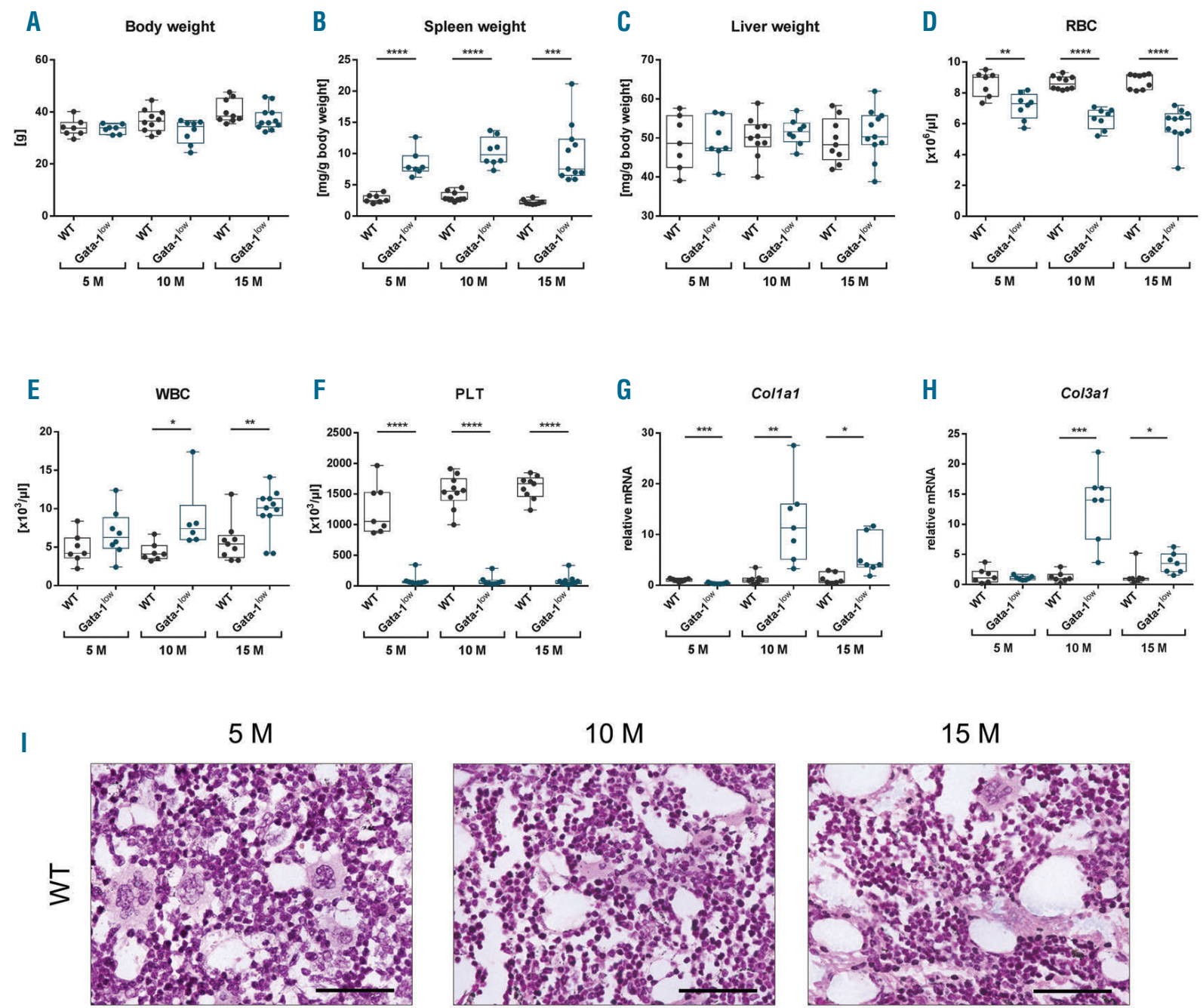

$10 \mathrm{M}$
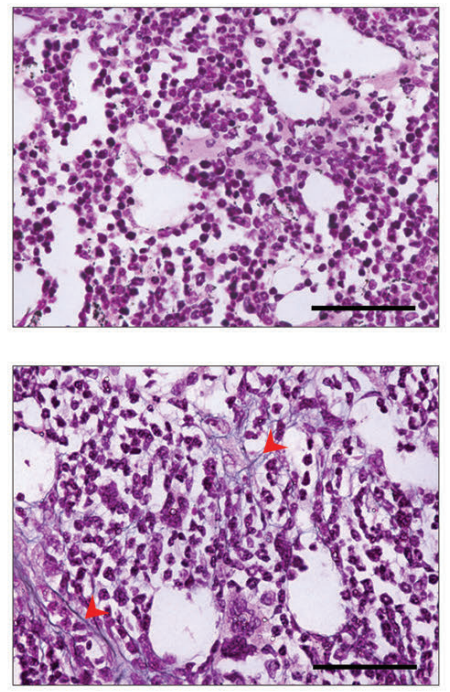

$15 \mathrm{M}$
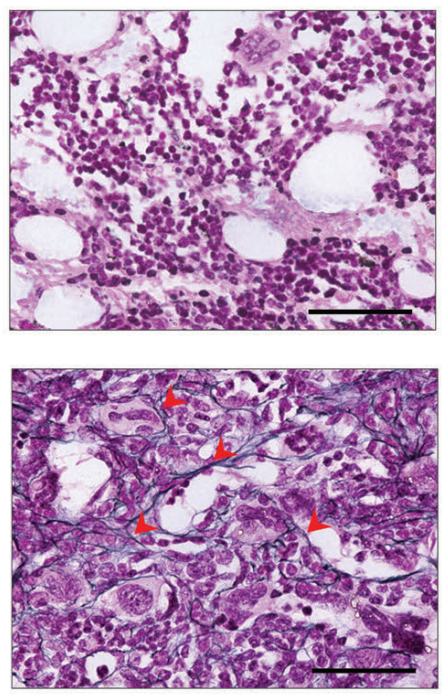

Figure 1. Characteristics of the Gata- $1^{\text {low }}$ mouse model for primary myelofibrosis at 5 months ( $\left.5 \mathrm{M}\right)$, 10 months (10 M), and 15 months (15 M) of age. (A) Body weight of Gata-1 $1^{\text {low }}$ mice and age-matched wild-type (WT) controls. (B) Spleen weight per g body weight. (C) Liver weight per g body weight. (D) Red blood cell (RBC) counts. (E) White blood cell (WBC) counts. (F) Platelet (PLT) counts. (G) Quantitative polymerase chain reaction analyses of type I collagen Col1a1 and (H) type III collagen Col3a1. (I) Representative images of reticulum-stained femoral bone marrow showing increasing amount of reticulum fibers in the bone marrow of Gata-1 ${ }^{\text {low }}$ mice, indicated by red arrowheads. Scale bar $=50 \mu \mathrm{m} . \mathrm{n}=7-11$ mice per group. ${ }^{\star} P \leq 0.05, * * P \leq 0.01, * * * P \leq 0.001, * * * * P \leq 0.0001$ versus the control group by Student $t$-test. 
enrichment analyses (Figure 2C). Interestingly, genes implicated in PDGF binding (Pdgfa, Pdgfb, Pdap1, Pdgfra, Pdgfrb, Col1a1, Col1a2, Col2a1, Col3a1, Col4a1, Colsa1 and Colba1) were most over-represented within the up-regulated genes, followed by ECM structural constituents and genes referring to collagen binding.

\section{Expression dynamics of platelet-derived growth factor} signaling components during the development of myelofibrosis

Platelet-derived growth factors and their receptors are linked to bone marrow fibrosis, as was inferred from their increased expression in PMF patients. ${ }^{9,10}$ However, data addressing the expression dynamics during the develop- ment of myelofibrosis are sparse. In order to characterize the expression pattern of PDGF signaling components at different stages of bone marrow fibrosis, we analyzed gene expression of the ligand and receptor genes in the bone marrow of Gata- $1^{\text {low }}$ mice by quantitative PCR (qPCR). Here, we observed that gene expression of both receptor genes Pdgfra and Pdgfrb was highly induced in early fibrotic bone marrow from 10 -month-old Gata- $1^{\text {low }}$ mice and remained increased in overt fibrotic bone marrow of 15 -month-old Gata-1 ${ }^{\text {low }}$ mice (Figure $3 \mathrm{~A}$ and $\mathrm{B}$ ). Interestingly, $P d g f r b$ gene expression was significantly decreased in pre-fibrotic bone marrow of 5-month-old Gata- $1^{\text {low }}$ mice, as also detected for Colla1 gene expression. qPCR analyses of the ligand genes Pdgfa and Pdgfb
A

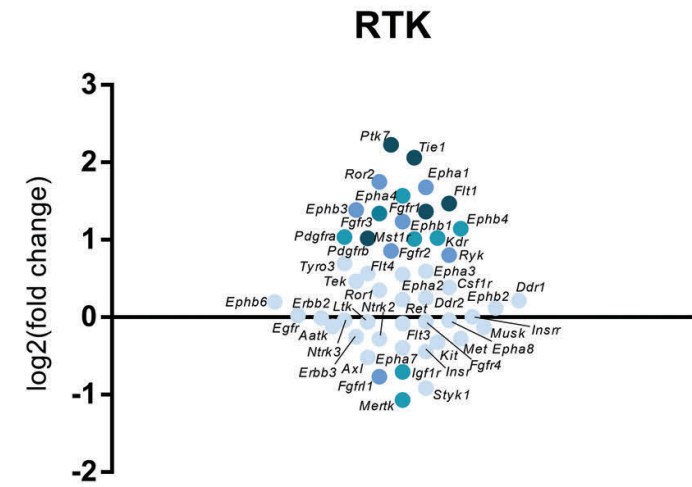

B

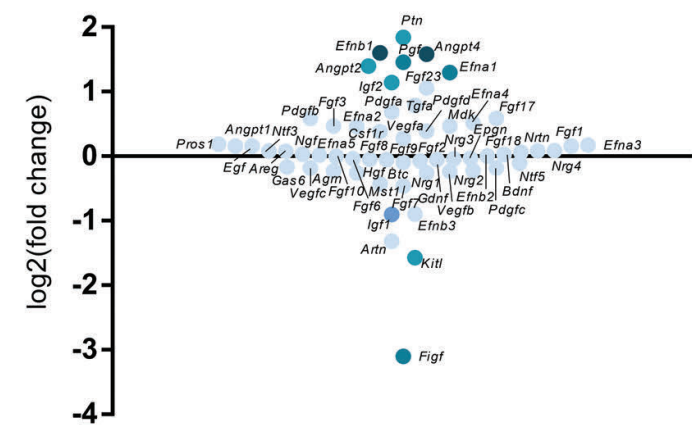

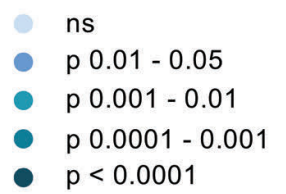

(1) ns

- p $0.01-0.05$

p $0.001-0.01$

- $p 0.0001-0.001$

- $\mathrm{p}<0.0001$

C

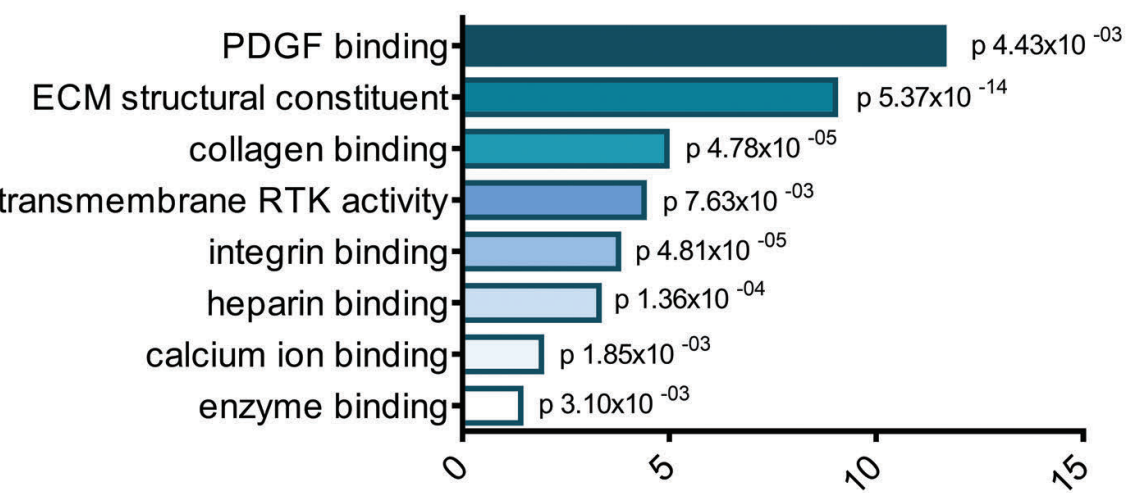

fold enrichment
Figure 2. RNA sequencing analyses showing gene expression of receptor tyrosine kinases (RTK), their ligands and gene ontology enrichment analy sis. Total RNA from femoral bone marrow of 10-month-old mice ( $n=3$ Gata-1 ${ }^{\text {1ow }}$ vs. $n=3$ wild-type mice) was analyzed. (A) Gene expression of RTK. (B) Gene expression of their cognate ligands. (C) Gene ontology enrichment analysis of over-expressed genes. 
revealed a major increase in ligand gene expression at the early fibrotic stage (Figure 3C and D). We again observed a decrease in $P d g f a$ gene expression in pre-fibrotic bone marrow, whereas $P d g f a$ gene expression was increased in early and overt fibrotic bone marrow. Pdgfb expression was significantly up-regulated only in early fibrotic bone marrow and remained at nearly baseline level in prefibrotic and overt fibrotic bone marrow of Gata- $1^{\text {low }}$ mice.

To validate the gene expression data, we further analyzed protein expression using an in situ proximity ligation assay (PLA) in a single recognition approach (Figure $3 \mathrm{E})$. By this method, two oligonucleotide-coupled secondary antibodies (PLA probes) detect a single primary antibody. Through ligation, oligonucleotides are joined to a circle when in close proximity and serve as template for polymerization. A polymerase replicates the DNA circles and a concatemeric product is generated. Fluorescentlylabeled nucleotides enable the detection of a rolling circle product (RCP), which can be visualized and quantified as a distinct fluorescent dot. ${ }^{17,18}$ Corresponding negative controls, positive controls, and results from the quantitative analyses by proximity ligation assay (PLA) in comparison to quantified protein expression data acquired from multiplex staining are shown in Online Supplementary Figures S1-S3. Although we observed a heterogenic protein expression in Gata- $1^{\text {low }}$ mice, there was a steady increase in PDGF receptor protein expression during the development of myelofibrosis (Figure 3F and G; original PLA images are shown in Online Supplementary Figures S4). When analyzing PDGF-A and PDGF-B protein expression by single recognition PLA, we again observed high heterogeneity among age-matched Gata- $1^{\text {low }}$ mice. We did not detect a significant increase in PDGF-A expression, while PDGF-B protein expression was significantly increased in overt fibrotic bone marrow of 15 -month-old Gata- $1^{\text {low }}$ mice (Figure $3 \mathrm{H}$ and I, original PLA images are shown in Online Supplementary Figures S4).

To visualize the cell type-specific expression of the PDGF signaling components, we performed multiplexed staining of PDGF signaling components in the bone marrow (Figure 4). We observed PDGFR $\alpha$ expression predominantly in megakaryocytes, whereas PDGFR $\beta$
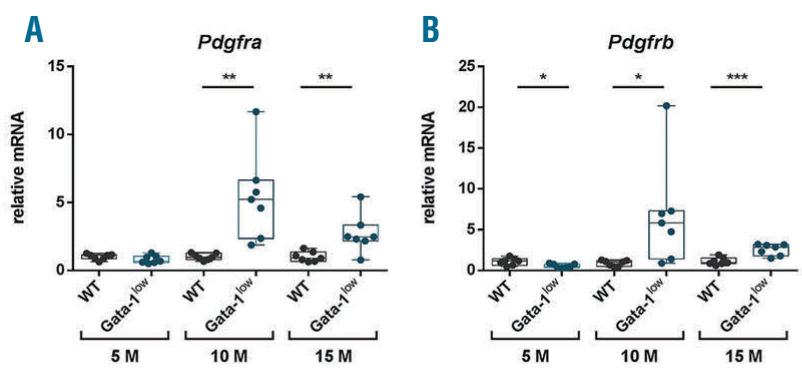

E

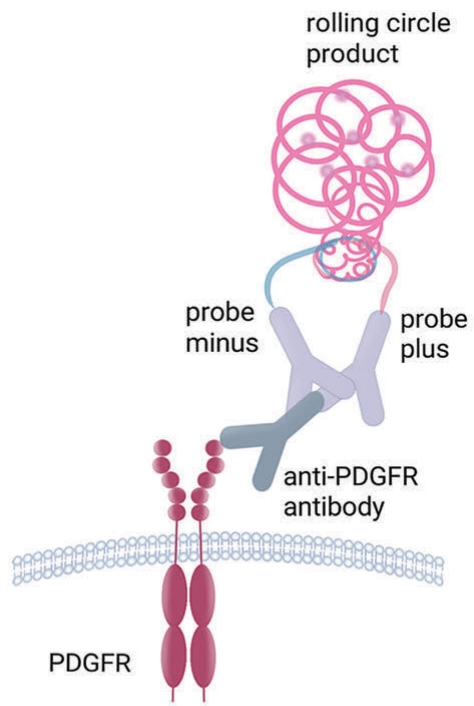

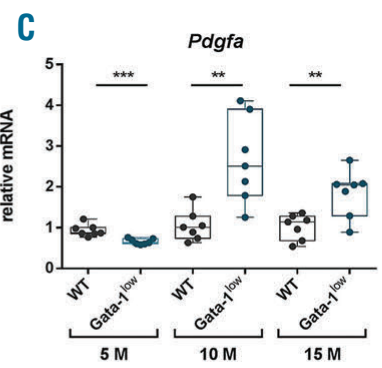

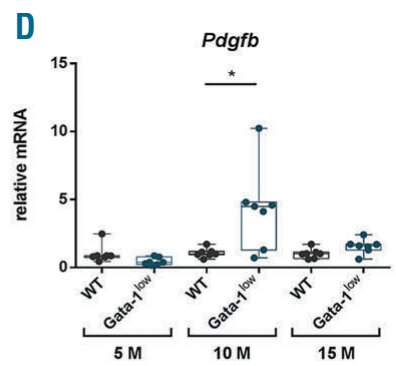

$\mathrm{F}$
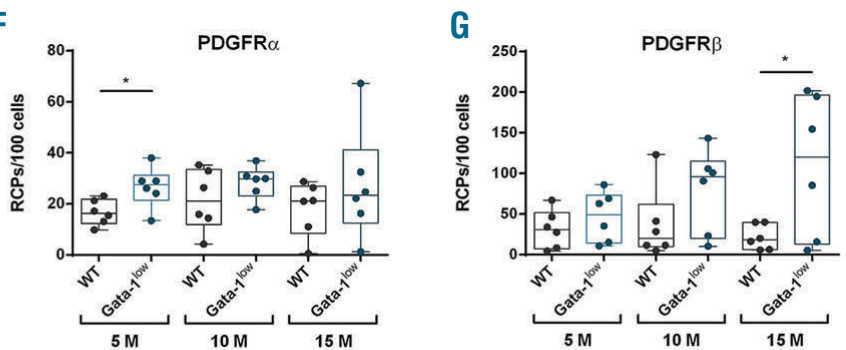

H

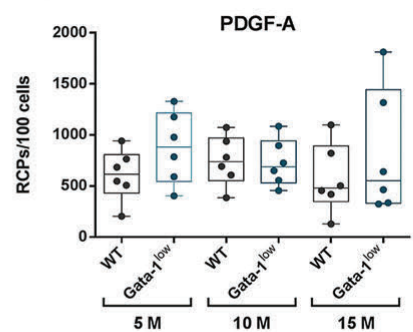

I

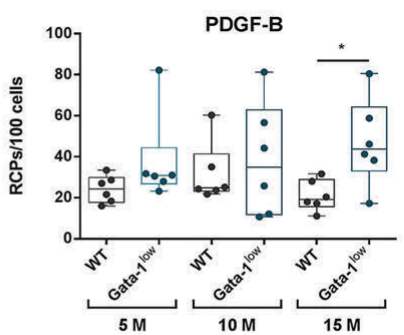

Figure 3. Expression of platelet-derived growth factors (PDGF) and their receptors in the bone marrow of Gata- $1^{\text {low }}$ mice at 5 months ( 5 M), 10 months (10 M) and 15 months (15 M) of age. (A) Quantitative polymerase chain reaction (qPCR) analyses of Pdgfra, (B) Pdgfrb, (C) Pdgfa, and (D) Pdgfb in the bone marrow of Gata-1 $1^{\text {low }}$ mice and age-matched wild-type (WT) controls. $n=7$ mice per group. (E) Illustration of a single recognition proximity ligation assay (PLA) as a sensitive means to quantify protein expression in situ. (F) Quantitative analyses of PDGFR $\alpha$, (G) PDGFR $\beta$, (H) PDGF-A, and (I) PDGF-B protein expression by single recognition PLA in the bone marrow of Gata- $1^{\text {low }}$ mice and age-matched WT controls. $n=6$ mice per group. 3170-7935 nucleated cells per mouse were analyzed for the presence of rolling circle products $(\mathrm{RCP}) . * P \leq 0.05, * * P \leq 0.01, * * * P \leq 0.001$ versus the control group by Student $t$-test. 


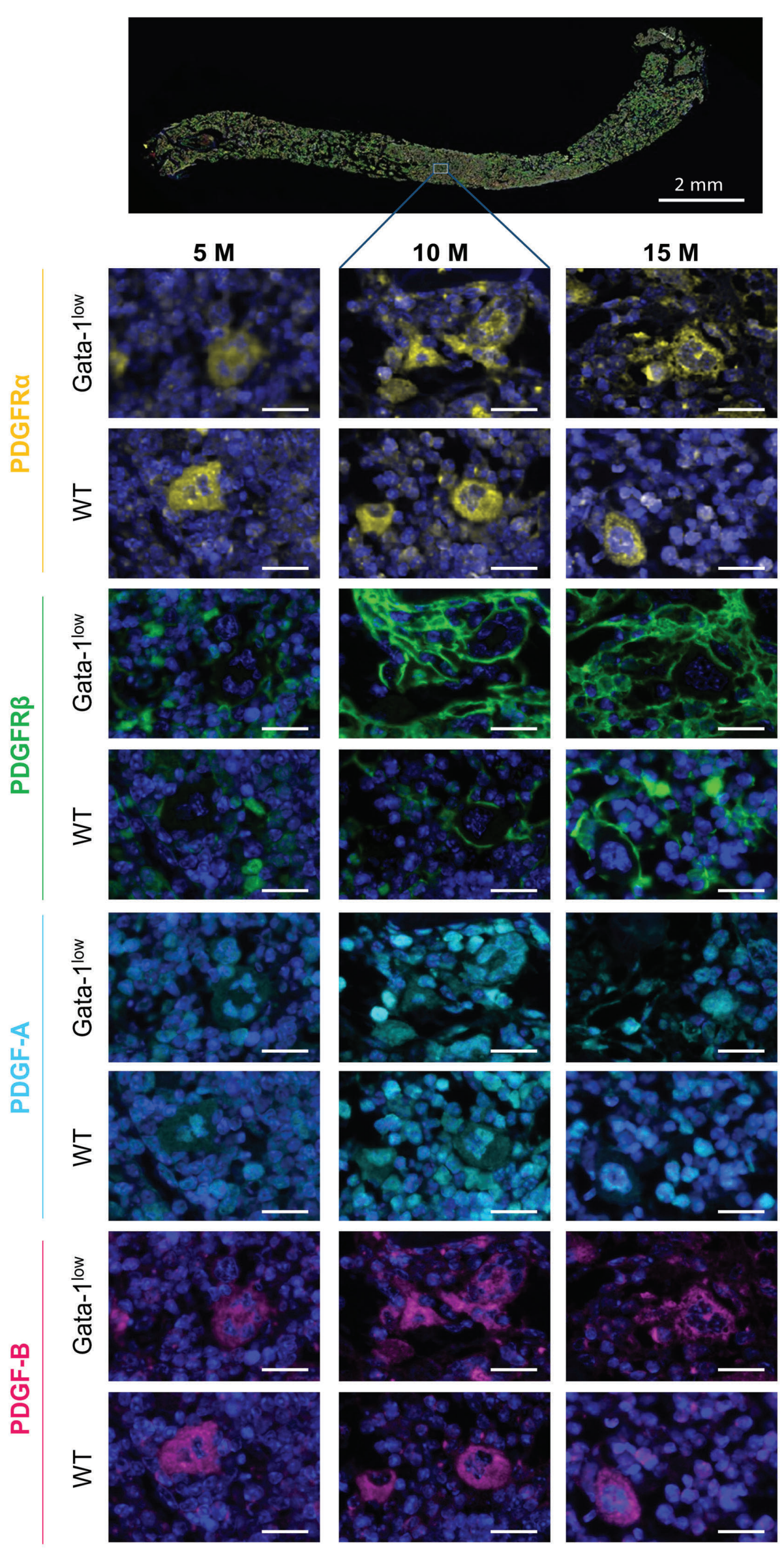

Figure 4. Multiplex staining of platelet-derived growth factors (PDGF) and their receptors in the bone marrow of Gata-1 $1^{\text {low }}$ mice at 5 months ( $5 \mathrm{M}), 10$ months ( $10 \mathrm{M})$ and

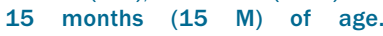
Representative images showing femoral bone marrow of Gata-1 $1^{\text {low }}$ mice and wild-type (WT) control mice stained for PDGF receptor $\alpha$ (PDGFR $\alpha$, yellow), PDGF receptor $\beta$ (PDGFR $\beta$, green), PDGF-A (cyan) and PDGF-B (magenta). 10 images at 40x magnification were acquired within the femoral bone marrow for each group. Nuclei were counterstained with DAPI (blue), scale bars in lower panels $=20 \mu \mathrm{m}$. 
marked spindle-shaped stromal cells in early and overt fibrotic bone marrow of Gata-1 $1^{\text {low }}$ mice. Staining of the ligand PDGF-A showed expression in a wide variety of different hematopoietic cells, whereas PDGF-B mainly derived from megakaryocytes, suggesting a paracrine effect of PDGF-B on PDGFR $\beta$ in bone marrow fibrosis.

Whereas megakaryocyte dysplasia and proliferation is a defining feature of PMF, fibroblast proliferation leading to a progressive fibrosis is the key pathological aspect of PMF. Given the distinct expression of PDGFR $\beta$ in stromal cells of early fibrotic and fibrotic bone marrow, we further concentrated on the dynamics of PDGFR $\beta$ and its ligand PDGF-B.

\section{Ligand-activation and regulation of PDGFR $\beta$ during the development of myelofibrosis}

The increased protein expression of PDGFR $\beta$ and its ligand PDGF-B in overt myelofibrosis prompted us to investigate the interaction of both signaling components in situ. To analyze PDGFR $\beta$-PDGF-B binding, we performed a PLA using combined primary antibodies detecting PDGFR $\beta$ and PDGF-B (Figure 5A). The assay allows for the detection and quantification of signals and showed an increased interaction of receptor and ligand in overt fibrotic bone marrow of 15 -month-old Gata- ${ }^{1 \text { low }}$ mice (Figure 5B, original PLA images are shown in Online Supplementary Figures S5). This is in accordance with enhanced protein
A

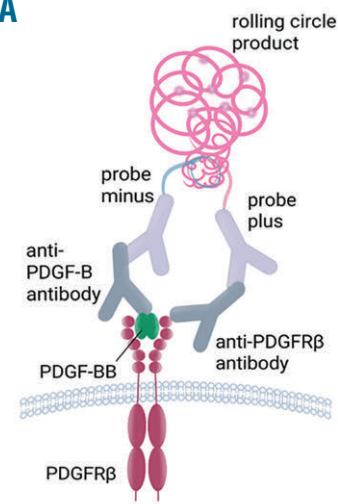

B

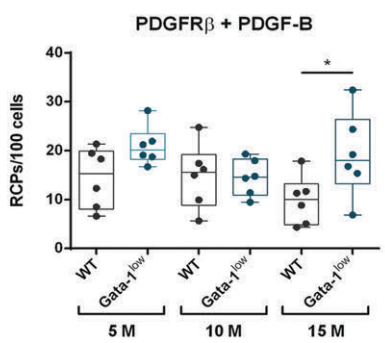

C

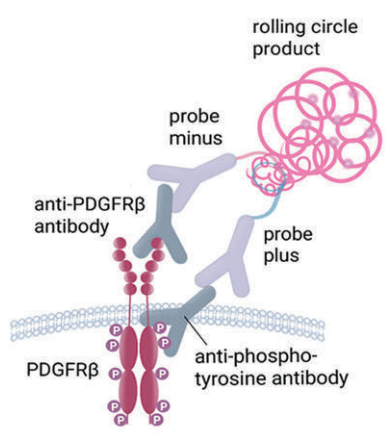

D

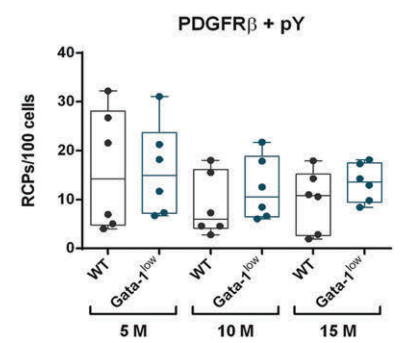

E

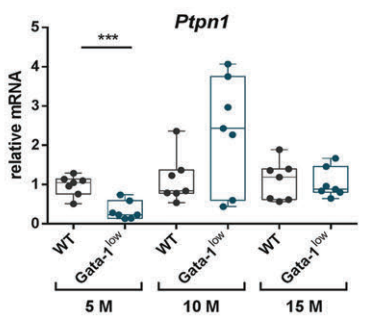

H

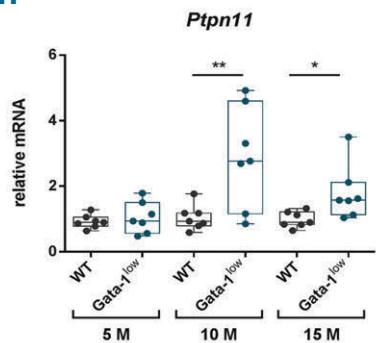

$\mathrm{F}$

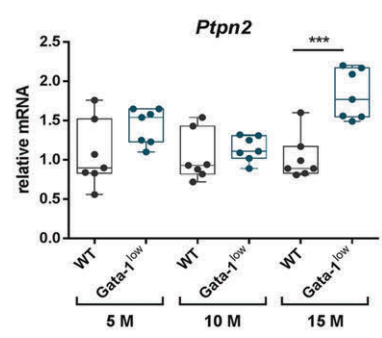

I

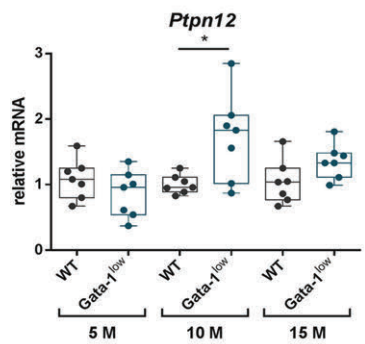

G

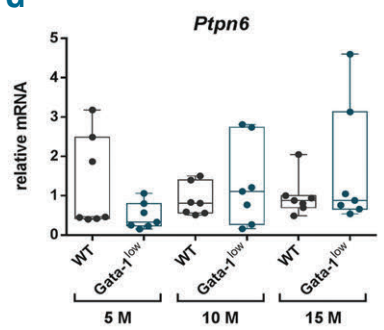

J

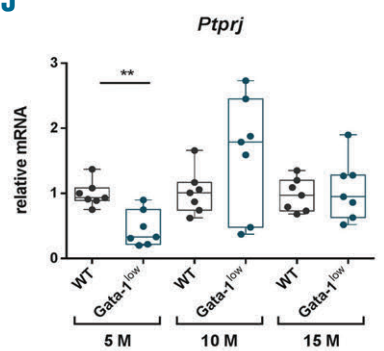

Figure 5. Interaction analysis of platelet-derived growth factor receptor $\beta$ (PDGFR $\beta$ ) with PDGF-B and PDGFR $\beta$ tyrosine phosphorylation in the bone marrow of Gata- $1^{\text {low }}$ mice at 5 months (5 M), 10 months $(10 \mathrm{M})$ and 15 months (15 M) of age. (A) Illustration of a proximity ligation assay (PLA) for the analysis of PDGFR $\beta$ PDGF-B interaction. (B) Quantitative analysis of PDGFR $\beta$-PDGF-B interaction by PLA in the bone marrow of Gata-1 ${ }^{\text {low }}$ mice and age-matched wild-type (WT) controls. (C) Illustration of a PLA for the analysis of PDGFR $\beta$ tyrosine phosphorylation. (D) Quantitative analysis of PDGFR $\beta$ tyrosine phosphorylation by PLA in the bone marrow of Gata- $1^{\text {low }}$ mice and age-matched WT controls, $n=6$ mice per group, 2670-9001 nucleated cells per mouse were analyzed for the presence of rolling circle products

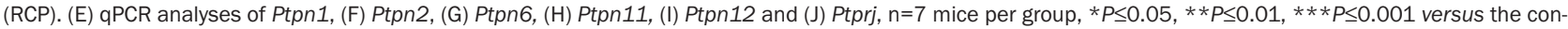
trol group by Student $t$-test. 
expression of both PDGFR $\beta$ and PDGF-B in the bone marrow of 15 -month-old Gata-1 $1^{\text {low }}$ mice and suggests an increased activation of intracellular signaling in the overt fibrotic stage. To further analyze the activation status of the receptor, we applied a PLA combining PDGFR $\beta$ and phosphotyrosine-targeting primary antibodies to analyze PDGFR $\beta$ phosphorylation in situ (Figure 5C). Surprisingly, we did not observe increased PDGFR $\beta$ tyrosine phosphorylation at any stage of myelofibrosis in Gata- $1^{\text {low }}$ mice (Figure 5D, original PLA images are shown in Online Supplementary Figure 5). To verify these results, we per- formed both an enzyme-linked immunosorbent assay (ELISA) with isolated protein from fresh frozen material and in situ staining for PDGFR $\beta$ phosphorylation at $Y^{751}$. In line with the PLA data, no differences in tyrosine phosphorylation were detectable between the WT animals and Gata-1 $1^{\text {low }}$ mice at all ages (data not shown). These results suggest the presence of further counter-regulatory mechanisms, such as PTP. Therefore, to investigate the contribution of the different PTP regarding the PDGFR $\beta$ phosphorylation status, we screened RNAseq data from 10-month-old Gata-1 ${ }^{\text {low }}$ mice and WT controls for differ-
A
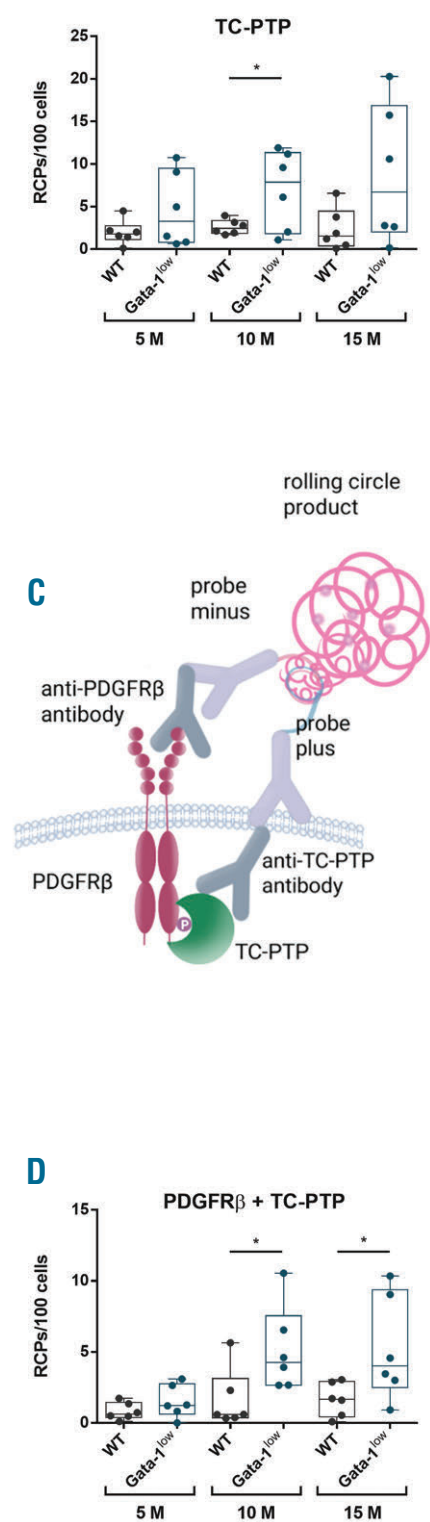

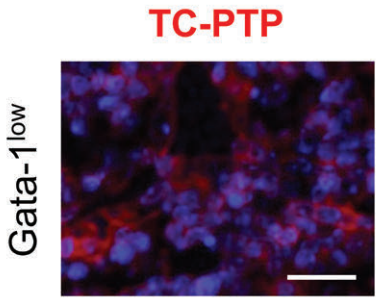

$\sum_{\infty}$
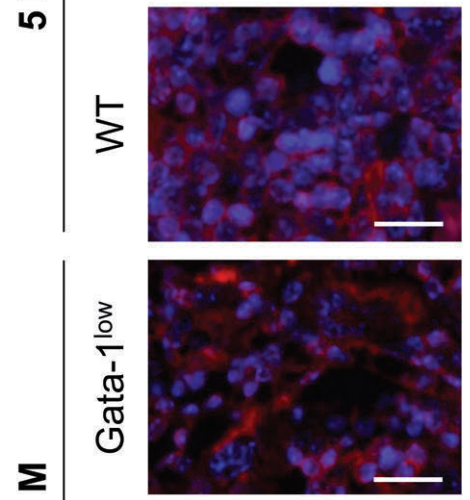

으
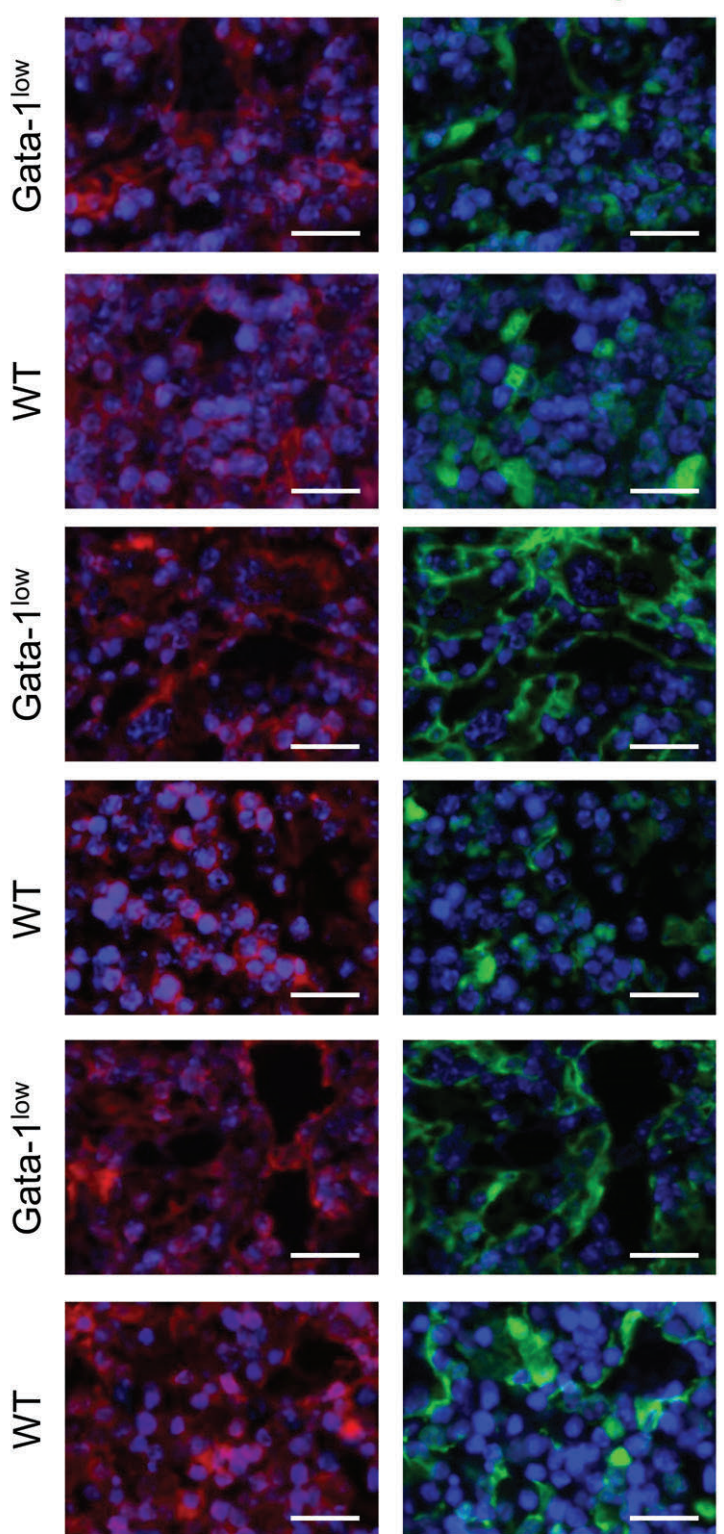

merge
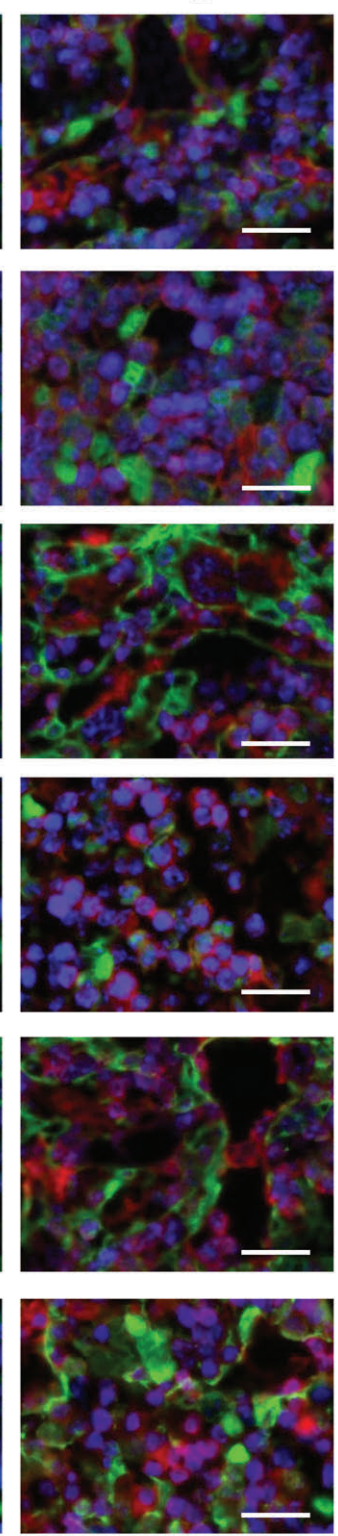

Figure 6. Expression and interaction dynamics of the phosphatase T-cell protein tyrosine phosphatase (TC-PTP) and platelet-derived growth factor receptor $\beta$ (PDGFR $\beta$ ) in the bone marrow of Gata- $1^{\text {low }}$ mice at 5 months (5 M), 10 months (10 M) and 15 months (15 M) of age. (A) Quantitative analyses of TC-PTP protein expression by single recognition proximity ligation assay (PLA) in the bone marrow of Gata-1 ${ }^{\text {low }}$ mice and age-matched wild-type (WT) controls. (B) Representative images showing femoral bone marrow of Gata- $1^{\text {low }}$ mice stained for PDGF receptor $\beta$ (PDGFR $\beta$, green) and TC-PTP (red). Nuclei were counterstained with DAPI (blue), scale bars $=20 \mathrm{~mm}$. (C) Illustration of a PLA for the analysis of PDGFR $\beta$-TC-PTP interaction. (D) Quantitative analysis of PDGFR $\beta$-TC-PTP interaction by PLA in the bone marrow of Gata- $1^{\text {low }}$ mice and age-matched WT controls, $n=6$ mice per group. 4051-9101 nucleated cells per mouse were analyzed for the presence of rolling circle products (RCP). ${ }^{*} P \leq 0.05$ versus the control group by Student $t$-test. 
ential expression of all classical PTP and the dual-specific phosphatase Pten. These analyses included the six PTP which are known to target PDGFR $\beta .{ }^{19-22}$ However, Ptpn1 (encoding PTP1B), Ptpn2 (encoding TC-PTP), Ptpn6 (encoding SHP-1), Ptpn11 (encoding SHP-2), Ptpn12 (encoding PTP-PEST), and Ptpri (encoding DEP-1) were not differentially expressed in the bone marrow of early fibrotic, 10-month-old mice (data not shown). To complement these data with gene expression analyses for Gata- $1^{\text {low }}$ mice of all ages, and to overcome the small sample size ( $\mathrm{n}=3$ mice per group) in the RNAseq analyses, we performed qPCR analyses for Ptpn1, Ptpn2, Ptpn6, Ptpn11, Ptpn12, and Ptpri. Indeed, in the gene expression analyses we observed distinct expression dynamics among gene expression of these PTP (Figure 5E-J). Overall, the data generated by qPCR from early fibrotic bone marrow of 10-month-old Gata-1 $1^{\text {low }}$ mice displayed pronounced biological variances, possibly contributing to the lack of significance within the RNAseq data. Interestingly, Ptpn1 and Ptprj gene expression, analyzed by GPCR, showed decreased expression in pre-fibrotic bone marrow. There was an increased expression of Ptpn11 and Ptpn12 in early
A

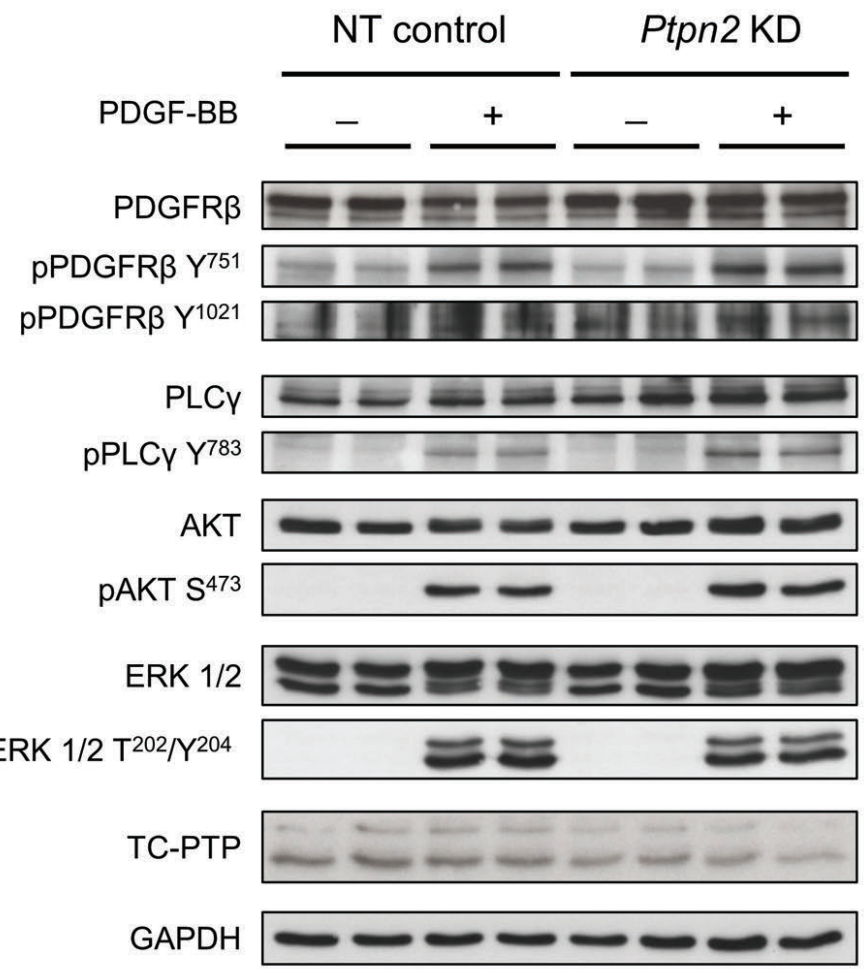

H

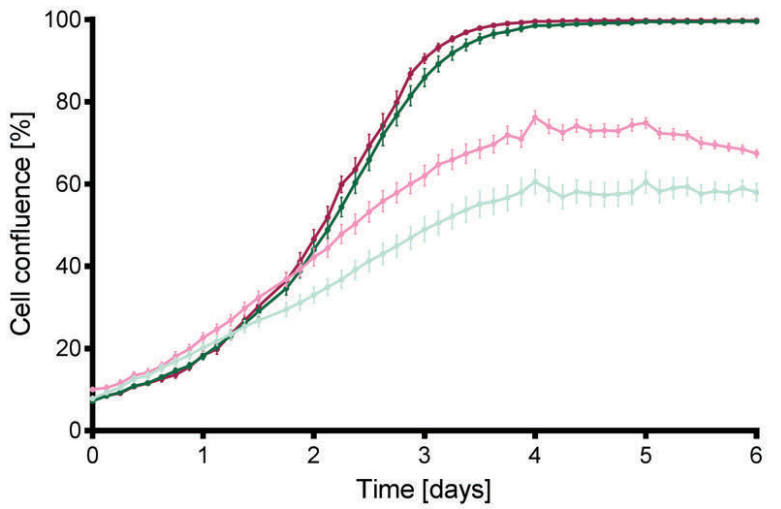

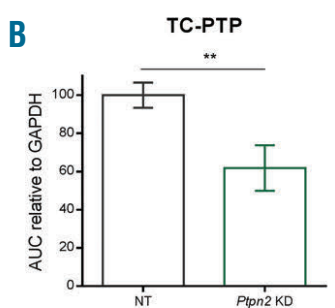

C
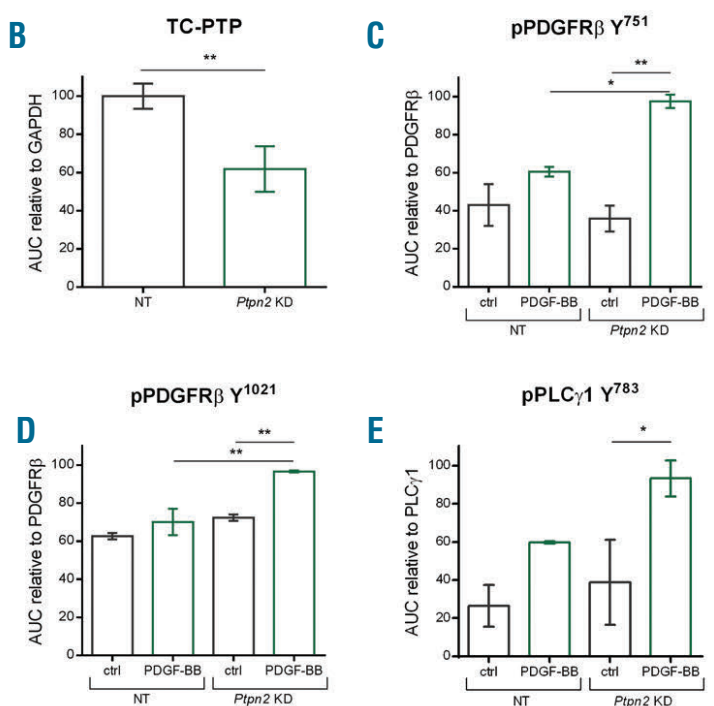

E
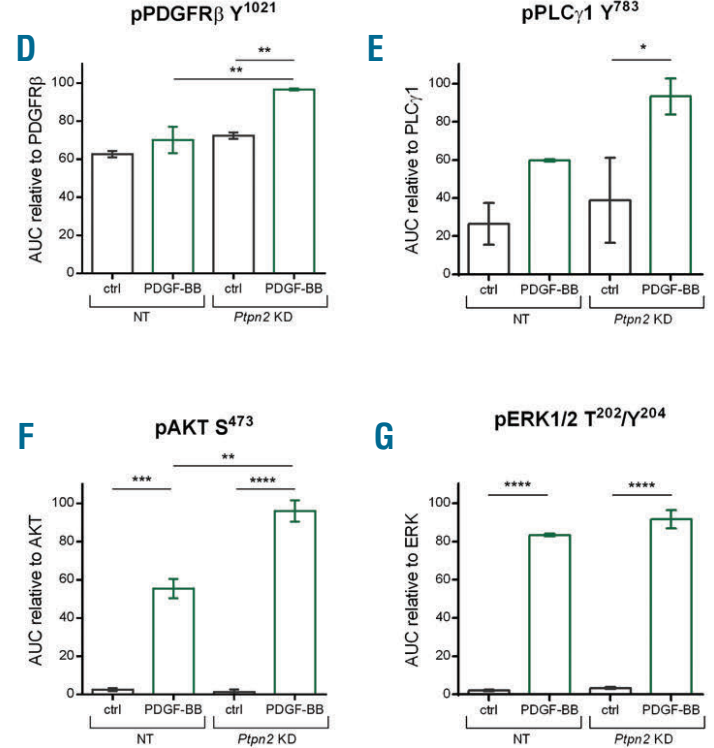

G

PERK1/2 $\mathrm{T}^{202} / \mathrm{Y}^{204}$

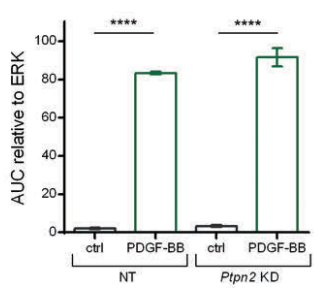

- NT, $1 \%$ FBS

- Ptpn $2 \mathrm{KD}, 1 \% \mathrm{FBS}$

$\rightarrow$ NT, $10 \%$ FBS

- Ptpn 2 KD, $10 \%$ FBS

Figure 7. Regulation of platelet-derived growth factor receptor $\beta$ (PDGFR $\beta$ ) signaling and proliferation of NIH-3T3 fibroblasts by T-cell protein tyrosine phosphatase (TC-PTP). (A) Immunoblot of Ptpn2 knock down (KD) and non-targeting (NT) control cells, untreated (-) and stimulated with 50 ng/mL PDGF-BB (+) for 5 minutes. (B) Densitometric analyses of TC-PTP, (C) pPDGFR $\beta Y^{751}$, (D) pPDGFR $\beta Y^{1021}$, (E) pPLC $11 Y^{783}$, (F) pAKT S ${ }^{473}$, (G) pERK1/2 T ${ }^{202} / Y^{204}$. (H) Proliferation curves of Ptpn2 KD and NT control cells cultured in $1 \%$ and $10 \%$ fetal bovine serum (FBS). ${ }^{*} P \leq 0.05, * * P \leq 0.01, * * * P \leq 0.001, * * * * P \leq 0.0001$ by analysis of variance with post hoc Tukey correction. 
fibrotic bone marrow. In overt fibrotic bone marrow, Ptpn2 and Ptpn11 gene expression was enhanced. Since TC-PTP (Ptpn2) has previously been ascribed an essential role in normal hematopoietic function, ${ }^{23,24}$ we further focused on PDGFR $\beta$ regulation by TC-PTP. Given the conclusive increase in Ptpn2 gene expression in overt fibrotic bone marrow, we next analyzed TC-PTP protein expression in the different fibrotic stages in Gata- $1^{\text {low }}$ mice. Quantitative analysis of TC-PTP protein expression by PLA confirmed an increased expression in early and overt myelofibrosis (Figure 6A, original PLA images are shown in Online Supplementary Figure S5). In addition, in situ imaging showed ubiquitous expression of TC-PTP in the bone marrow of Gata- $1^{\text {low }}$ mice (Figure $6 \mathrm{~B}$ ). TC-PTP staining was positive in a wide variety of hematopoietic cells, megakaryocytes and in spindle-shaped cell structures, raising the question if TC-PTP directly regulates PDGFR $\beta$ in bone marrow stromal cells. To determine the interaction of PDGFR $\beta$ and TC-PTP, we again applied the sensitive proximity ligation technique (Figure 6C) and detected increased interaction of PDGFR $\beta$ and TC-PTP in early and overt fibrotic bone marrow of Gata- $1^{\text {low }}$ mice (Figure $6 \mathrm{D}$, original PLA images are shown in Online Supplementary Figure S5).

\section{TC-PTP in PDGFR $\beta$ signaling and proliferation in fibroblasts in vitro}

Our findings of increased PDGFR $\beta$ and TC-PTP expression and interaction led us to further analyze the regulation of PDGFR $\beta$ by TC-PTP in fibroblasts in vitro. We knocked down Ptpn2 in NIH-3T3 fibroblasts and stimulated cells with PDGF-BB (Figure 7A). Transfection with Ptpn2-targeting siRNA resulted in a moderate but significant knockdown (KD) compared to cells transfected with non-targeting (NT) siRNA (Figure 7B). We observed a consecutive increase in PDGFR $\beta$ phosphorylation at $\mathrm{Y}^{751}$ and downstream AKT signaling in Ptpn2 KD cells (Figure 7C and F). However, there was no substantial effect on downstream ERK signaling (Figure 7G). PDGFR $\beta$ phosphorylation at $\mathrm{Y}^{1021}$, as well as downstream PLC $\gamma$ activation, was increased in Ptpn2 KD cells (Figure 7D and E). We further monitored proliferation of Ptpn2 KD cells (Figure $7 \mathrm{H}$ ) and did not find any evident differences in proliferation in cells cultured in complete growth medium containing $10 \%$ fetal bovine serum (FBS). However, when cells were exposed to serum-reduced medium (1\% FBS), Ptpn2 KD cells showed increased growth rates compared to NT control cells.

\section{Discussion}

In this study, we provide detailed analyses of the expression patterns of PDGFR $\beta$ signaling in the bone marrow of Gata- $1^{\text {low }}$ mice at different fibrotic disease stages using RNAseq, qPCR, in situ protein expression analyses, multiplex staining and PLA. Early and overt fibrotic bone marrow was characterized by increased expression of PDGF signaling components and overt fibrosis by an increase in PDGFR $\beta-P D G F-B$ interaction. Since PDGFR $\beta$ tyrosine phosphorylation levels were not increased, the regulation of PDGFR $\beta$ by PTP was investigated. Ptpn2 gene as well as TC-PTP protein expression was increased in fibrotic bone marrow of Gata- $1^{\text {low }}$ mice. Furthermore, enhanced PDGFR $\beta$-TC-PTP interaction was observed in early and overt myelofibrosis, potentially counteracting PDGFR $\beta$ phosphorylation. Likewise, Ptpn $2 \mathrm{KD}$ increased PDGFR $\beta$ tyrosine phosphorylation at $Y^{751}$ and $Y^{1021}$ and resulted in enhanced downstream AKT and PLC $\gamma 1$ signaling in fibroblasts. Furthermore, Ptpn2 KD cells showed a growth condition-dependent increase in expansion rate. Thus, while PDGF signaling is differentially regulated during PMF, PTP seem novel and so far unrecognized components in disease development. Previously not applied in bone marrow, PLA might add to diagnostics as a novel technique.

Intense efforts have been made to understand the mechanisms leading to PMF, focusing on genetic analysis. However, the PMF-associated driver mutations leading to aberrant activation of JAK-STAT signaling are not unique to PMF but also occur in other MPN, namely essential thrombocythemia and polycythemia vera. Although one of the driver mutations is sufficient to induce PMF in patients, they are often accompanied by other mutations and epigenetic changes. ${ }^{25,26}$ However, there is still no distinct molecular marker available for the respective MPN, emphasizing that the underlying mechanisms directing the different MPN are not yet understood. The discovery of JAK-STAT-associated mutations led to the development of JAK inhibitors and since its US Food and Drug Administration approval in 2011, the JAK1/2 inhibitor ruxolitinib has become part of combined standard therapy for PMF patients. Long-term treatment with ruxolitinib reduces spleen size and prolongs the overall survival of PMF patients. ${ }^{27}$ However, there is no improvement or reversal of bone marrow fibrosis. Furthermore, efficacy of ruxolitinib is limited by drug resistance, ${ }^{28}$ and JAK inhibition does not abrogate clonal proliferation. ${ }^{29}$ Recently, PDGFR $\alpha$ signaling was shown to remain active despite JAK2 inhibition in vivo and is a cell-intrinsic bypass for maintaining downstream ERK signaling upon ruxolitinib treatment. ${ }^{30}$ To date, allogeneic stem cell transplantation is the only curative treatment for PMF; however, transplantation is only suitable for a subset of high-risk patients and limited by comorbidities and donor availability. ${ }^{31,32}$

Gata- $1^{\text {low }}$ mice were characterized by a marked thrombocytopenia, splenomegaly and progressive anemia starting before 5 months of age. Enrichment analyses of RNAseq data from early fibrotic bone marrow of Gata- $1^{\text {low }}$ mice revealed that genes implicated in PDGF binding are most over-represented within the up-regulated genes. We further observed enhanced PDGFR $\beta$ and PDGF-B protein expression at 15 months of age, along with an increase in PDGFR $\beta-P D G F-B$ interaction, analyzed by PLA.

However, we did not detect an increase in PDGFR $\beta$ tyrosine phosphorylation in the bone marrow of Gata- $1^{\text {low }}$ mice. This observation raised the question as to whether other mechanisms are involved in the regulation of the receptor in fibrotic bone marrow. Since a number of PTP have been identified, which site-selectively dephosphorylate PDGFR $\beta$, namely PTP1B, TC-PTP, SHP-1, SHP-2, PTPPEST and DEP-1, ${ }^{19-22}$ an increased expression of these PTP might be responsible for the absence of an increased PDGFR $\beta$ phosphorylation. Indeed, our data showed distinct dynamics in gene expression of these PTP.

We observed decreased Ptpn1 and Ptprj gene expression in pre-fibrotic bone marrow in Gata- $1^{\text {low }}$ mice. The relevance of these PTP as potential diagnostic markers could be validated in a translational clinical approach. In contrast, Ptpn2, Ptpn11 and Ptpn12 showed an increased gene 
expression during the development of myelofibrosis in Gata- $1^{\text {low }}$ mice. Ptpn11, encoding the phosphatase SHP-2, has been described as positive regulator by mediating binding of Grb2 and thus promotes ERK signaling. Therefore, interpretation of enhanced Ptpn11 expression is complex and context dependent. Ptpn12, coding for PTPPEST, has previously been implicated in dendritic cell migration and macrophage fusion. ${ }^{35,36}$ However, data on Ptpn12 involvement in bone marrow malignancies are scarce. Ptpnz and its gene product TC-PTP, however, play a pivotal role in normal hematopoietic and stromal cell function, as emphasized by studies using TC-PTP deficient mice. Homozygous mice die 3-5 weeks after birth with severe defects in hematopoiesis. ${ }^{24}$ Transplantation experiments further suggest that TC-PTP knockout leads to changes in the bone marrow microenvironment, which impede normal HSC function. ${ }^{24}$ This supports the findings in our study, which indicate that TC-PTP expression in bone marrow stromal cells and the interaction with PDGFR $\beta$ might be important mediators of changes in the bone marrow microenvironment during the development of myelofibrosis. Ptpn2 gene expression was increased in overt fibrotic bone marrow of Gata- $1^{\text {low }}$ mice, implicating the importance of TC-PTP also with regard to PDGFR $\beta$ regulation. TC-PTP is ubiquitously expressed but shows strong expression in cells of the different hematopoietic lineages. We observed TC-PTP expression in hematopoietic cells, megakaryocytes as well as in stromal cells within the bone marrow. In addition to PDGFR $\beta$, TC-PTP dephosphorylates EGFR and JAK-STAT signaling components. ${ }^{37,38}$ Importantly, there are several lines of evidence showing TC-PTP is involved in a number of bone marrow alterations. ${ }^{23,24,39}$ Ultimately, a pharmacological approach with a highly efficient modulator of TC-PTP activity in vivo will be needed to provide evidence for TC-PTP contribution to the pathogenesis of myelofibrosis, which is a limitation of our study. A direct pharmacological intervention of TC-PTP in vivo, however, is not currently available. Consistent with other studies, ${ }^{21}$ we were able to show a counter-regulation of PDGFR $\beta$ by TC-PTP in fibroblasts in vitro. Ptpn $2 \mathrm{KD}$ resulted in enhanced PDGFR $\beta$ tyrosine phosphorylation at $\mathrm{Y}^{751}$, which serves as a binding site for PI3K. ${ }^{40}$ Conclusively, we detected an increase in downstream AKT activation as a central mediator of cell proliferation. PDGFR $\beta$ phosphorylation also activates Ras and downstream ERK signaling; ${ }^{41}$ however, we did not observe increased ERK signaling in Ptpn2 KD cells. Ptpn2 KD further led to increased PDGFR $\beta$ tyrosine phosphorylation at $\mathrm{Y}^{1021}$, resulting in enhanced downstream PLC $\gamma 1$ activation, suggesting a possible role of downstream protein kinase $\mathrm{C}$ and $\mathrm{Ca}^{2+}$ signaling.

We observed that Ptpn2 KD fibroblasts cultured in com- plete growth medium containing 10\% FBS did not have an apparent superiority in proliferation. However, we detected increased growth rates in Ptpn2 KD cells exposed to reduced-serum media containing $1 \% \mathrm{FBS}$. This suggests that under conditions of high abundance of growth factor differences in proliferation in Ptpn2 KD cells are abolished, while these are apparent during serum-deprivation. Other studies using murine skin cancer models showed that TCPTP controls proliferation and survival via AKT and STAT3 activation. ${ }^{42,43}$ Furthermore, emphasizing the role of TC-PTP in hematopoietic cells, TC-PTP controls T-cell proliferation. ${ }^{44}$ Our data, based on a moderate $\mathrm{KD}$, indicate that more discrete changes in TC-PTP expression controls cell growth mainly when the availability of growth components is limited.

In this study, we applied a PLA as a novel technique to analyze in situ alterations in bone marrow disease progression. The data acquired by PLA are generally in good agreement with our data acquired by multiplex staining, as another antibody-based method. While some discrepancies remained, further optimization regarding this tissue-specific approach are desired. This refers in particular to fluorescent signals, which are distinct from the clearly recognizable RCP. Those are most likely not ascribed to primary antibody binding but are caused by binding of oligonucleotides conjugated to secondary antibodies (PLA probes). Indeed, such signals have also been observed using DNA probes in in situ hybridization (FISH) approaches on bone marrow tissue and are associated with eosinophils. ${ }^{45}$ Future studies in patient material are warranted to evaluate the applicability of the PLA as a diagnostic tool in early disease stages, to monitor disease progression and response to JAK inhibition. These analytical methods should carefully consider pre-analytic processing such as specific decalcification protocols and archiving conditions for long-term storage of specimens.

In summary, PDGF signaling components display major alterations in bone marrow fibrosis. While PDGF and their cognate receptors are dynamically regulated, PTP represent previously unrecognized contributors that control PDGF signaling in myelofibrosis. As this study focused on PDGFR $\beta$-TC-PTP interaction within the bone marrow in situ microenvironment, future examination of PDGFR $\beta$ regulation by TC-PTP in primary stromal cells from mouse models and from PMF patients will help to elucidate the precise role of TC-PTP in the development of bone marrow disease.

\section{Funding}

The authors would like to thank the Stiftung für Pathobiochemie und Molekulare Diagnostik for funding to KK and the Sonnenfeld Stiftung (Berlin) for a doctoral scholarship to FK.

\section{References}

1. O'Sullivan JM, Harrison CN. Myelofibrosis: clinicopathologic features, prognosis, and management. Clin Adv Hematol Oncol. 2018;16(2):121-131.

2. Tefferi A. Pathogenesis of myelofibrosis with myeloid metaplasia. J Clin Oncol. 2005;23(33):8520-8530.

3. Kleppe M, Kwak M, Koppikar P, et al. JAK-
STAT pathway activation in malignant and nonmalignant cells contributes to MPN pathogenesis and therapeutic response. Cancer Discov. 2015;5(3):316-331.

4. Baxter EJ, Scott LM, Campbell PJ, et al. Acquired mutation of the tyrosine kinase JAK2 in human myeloproliferative disorders. Lancet. 2005;365(9464):1054-1061.

5. Pikman $Y$, Lee $B H$, Mercher $T$, et al. MPLW515L is a novel somatic activating mutation in myelofibrosis with myeloid metaplasia. PLoS Med. 2006;3(7):e270.

6. Klampfl T, Gisslinger H, Harutyunyan AS et al. Somatic mutations of calreticulin in myeloproliferative neoplasms. N Engl J Med. 2013;369(25):2379-2390.

7. Caenazzo A, Pietrogrande F, Polato G, et al. Changes in the mitogenic activity of platelet-derived growth factor(s) in patients with myeloproliferative disease. Acta Haematol. 1989;81(3):131-135.

8. Reilly JT. Pathogenesis of idiopathic 
myelofibrosis: role of growth factors. J Clin Pathol. 1992;45(6):461-464.

9. Bock O, Loch G, Busche G, von Wasielewski R, Schlue J, Kreipe H. Aberrant expression of platelet-derived growth factor (PDGF) and PDGF receptoralpha is associated with advanced bone marrow fibrosis in idiopathic myelofibrosis. Haematologica. 2005;90(1):133-134.

10. Bedekovics J, Kiss A, Beke L, Karolyi K, Mehes G. Platelet derived growth factor receptor-beta (PDGFRbeta) expression is limited to activated stromal cells in the bone marrow and shows a strong correlation with the grade of myelofibrosis. Virchows Arch. 2013:463(1):57-65.

11. Fredriksson L, Li H, Eriksson U. The PDGF family: four gene products form five dimeric isoforms. Cytokine Growth Factor Rev. 2004;15(4):197-204.

12. Heldin CH, Ostman A, Ronnstrand L. Signal transduction via platelet-derived growth factor receptors. Biochim Biophys Acta. 1998;1378(1):F79-113.

13. Wickenhauser C, Hillienhof A, Jungheim K, et al. Detection and quantification of transforming growth factor beta (TGF-beta) and platelet-derived growth factor (PDGF) release by normal human megakaryocytes. Leukemia. 1995;9(2):310-315.

14. Heldin $\mathrm{CH}$, Westermark B. Mechanism of action and in vivo role of platelet-derived growth factor. Physiol Rev. 1999;79(4): 1283-1316.

15. Bonner JC. Regulation of PDGF and its receptors in fibrotic diseases. Cytokine Growth Factor Rev. 2004;15(4):255-273

16. Vannucchi AM, Bianchi L, Cellai C, et al. Development of myelofibrosis in mice genetically impaired for GATA-1 expression (GATA-1(low) mice). Blood. 2002; 100(4):1123-1132.

17. Soderberg O, Leuchowius KJ, Gullberg M, et al. Characterizing proteins and their interactions in cells and tissues using the in situ proximity ligation assay. Methods. 2008;45(3):227-232.

18. Fredriksson S, Gullberg M, Jarvius J, et al. Protein detection using proximity-dependent DNA ligation assays. Nat Biotechnol. 2002;20(5):473-477.

19. Klinghoffer RA, Kazlauskas A. Identification of a putative Syp substrate, the PDGF beta receptor. J Biol Chem. 1995; 270(38):22208-22217.

20. Markova B, Herrlich P, Ronnstrand L, Bohmer FD. Identification of protein tyrosine phosphatases associating with the PDGF receptor. Biochemistry. 2003; 42(9):2691-2699.

21. Persson C, Savenhed C, Bourdeau A, et al. Site-selective regulation of platelet-derived growth factor beta receptor tyrosine phosphorylation by T-cell protein tyrosine phosphatase. Mol Cell Biol. 2004;
24(5):2190-2201.

22. Kappert K, Paulsson J, Sparwel J, et al. Dynamic changes in the expression of DEP1 and other PDGF receptor-antagonizing PTPs during onset and termination of neointima formation. FASEB J. 2007; 21(2):523-534

23. Wiede F, Chew SH, van Vliet C, et al. Strain-dependent differences in bone development, myeloid hyperplasia, morbidity and mortality in ptpn2-deficient mice. PLoS One. 2012;7(5):e36703.

24. You-Ten KE, Muise ES, Itie $A$, et al. Impaired bone marrow microenvironment and immune function in $\mathrm{T}$ cell protein tyrosine phosphatase-deficient mice. J Exp Med. 1997;186(5):683-693

25. Vannucchi AM, Lasho TL, Guglielmelli P, et al. Mutations and prognosis in primary myelofibrosis. Leukemia. 2013;27(9):18611869

26. Martinez-Calle N, Pascual M, Ordonez R, et al. Epigenomic profiling of myelofibrosis reveals widespread DNA methylation changes in enhancer elements and ZFP36L1 as a potential tumor suppressor gene epigenetically regulated. Haematologica. 2019, 104(8): $1572-1579$.

27. Verstovsek S, Mesa RA, Gotlib J, et al. Long-term treatment with ruxolitinib for patients with myelofibrosis: 5 -year update from the randomized, double-blind, placebo-controlled, phase 3 COMFORT-I trial. J Hematol Oncol. 2017;10(1):55

28. Harrison CN, Vannucchi AM, Kiladjian JJ et al. Long-term findings from COMFORTII, a phase 3 study of ruxolitinib vs best available therapy for myelofibrosis. Leukemia. 2016;30(8):1701-1707.

29. Deininger M, Radich J, Burn TC, Huber R, Paranagama D, Verstovsek $S$. The effect of long-term ruxolitinib treatment on JAK2p.V617F allele burden in patients with myelofibrosis. Blood. 2015;126(13):15511554

30. Stivala S, Codilupi T, Brkic $\mathrm{S}$, et al. Targeting compensatory MEK/ERK activation increases JAK inhibitor efficacy in myeloproliferative neoplasms. J Clin Invest. 2019;130:1596-1611.

31. Kroger NM, Deeg JH, Olavarria E, et al. Indication and management of allogeneic stem cell transplantation in primary myelofibrosis: a consensus process by an EBMT/ELN international working group. Leukemia. 2015;29(11):2126-2133.

32. McLornan DP, Yakoub-Agha I, Robin M, Chalandon Y, Harrison CN, Kroger N State-of-the-art review: allogeneic stem cell transplantation for myelofibrosis in 2019 Haematologica. 2019;104(4):659-668.

33. Ronnstrand L, Arvidsson AK, Kallin A, et al. SHP-2 binds to Tyr763 and Tyr1009 in the PDGF beta-receptor and mediates PDGFinduced activation of the Ras/MAP kinase pathway and chemotaxis. Oncogene. 1999, 18(25):3696-3702

34. Dance M, Montagner A, Salles JP, Yart A Raynal P. The molecular functions of Shp2 in the Ras/Mitogen-activated protein kinase (ERK1/2) pathway. Cell Signal. 2008; 20(3):453-459.

35. Rhee I, Davidson D, Souza CM, Vacher J, Veillette A. Macrophage fusion is controlled by the cytoplasmic protein tyrosine phosphatase PTP-PEST/PTPN12. Mol Cell Biol. 2013;33(12):2458-2469.

36. Rhee I, Zhong MC, Reizis B, Cheong C, Veillette A. Control of dendritic cell migration, T cell-dependent immunity, and autoimmunity by protein tyrosine phosphatase PTPN12 expressed in dendritic cells. Mol Cell Biol. 2014;34(5):888-899.

37. Pike KA, Tremblay ML TC-PTP and PTP1B: Regulating JAK-STAT signaling, controlling lymphoid malignancies. Cytokine. 2016:82:52-57.

38. Tiganis T, Bennett AM, Ravichandran KS, Tonks NK. Epidermal growth factor receptor and the adaptor protein p52Shc are specific substrates of T-cell protein tyrosine phosphatase. Mol Cell Biol. 1998, 18(3):1622-1634

39. Bourdeau A, Trop S, Doody KM, Dumont DJ, Tremblay ML. Inhibition of T cell protein tyrosine phosphatase enhances interleukin-18-dependent hematopoietic stem cell expansion. Stem Cells. 2013;31(2):293304.

40. Panayotou G, Bax B, Gout I, et al Interaction of the p85 subunit of PI 3-kinase and its N-terminal SH2 domain with a PDGF receptor phosphorylation site: structural features and analysis of conformational changes. EMBO J. 1992;11(12):4261-4272.

41. Kashishian A, Kazlauskas A, Cooper JA Phosphorylation sites in the PDGF receptor with different specificities for binding GAP and $\mathrm{PI} 3$ kinase in vivo. EMBO J 1992;11(4):1373-1382.

42. Lee H, Morales LD, Slaga TJ, Kim DJ. Activation of T-cell protein-tyrosine phosphatase suppresses keratinocyte survival and proliferation following UVB irradiation. J Biol Chem. 2015;290(1):13-24.

43. Lee H, Kim M, Baek M, et al. Targeted disruption of TC-PTP in the proliferative compartment augments STAT3 and AKT signaling and skin tumor development. Sci Rep. 2017;7:45077.

44. Wiede F, La Gruta NL, Tiganis T. PTPN2 attenuates T-cell lymphopenia-induced proliferation. Nat Commun. 2014;5:3073.

45. Patterson S, Gross J, Webster AD. DNA probes bind non-specifically to eosinophils during in situ hybridization: carbol chromotrope blocks binding to eosinophils but does not inhibit hybridization to specific nucleotide sequences. I Virol Methods. 1989;23(2):105-109. 